\title{
The number of failed components in a coherent working system when the lifetimes are discretely distributed
}

\author{
Krzysztof Jasiński ${ }^{1}$
}

Received: 10 August 2020 / Accepted: 30 March 2021 / Published online: 19 April 2021

(C) The Author(s) 2021

\begin{abstract}
In this paper, we study the number of failed components of a coherent system. We consider the case when the component lifetimes are discrete random variables that may be dependent and non-identically distributed. Firstly, we compute the probability that there are exactly $i, i=0, \ldots, n-k$, failures in a $k$-out-of- $n$ system under the condition that it is operating at time $t$. Next, we extend this result to other coherent systems. In addition, we show that, in the most popular model of independent and identically distributed component lifetimes, the obtained probability corresponds to the respective one derived in the continuous case and existing in the literature.
\end{abstract}

Keywords Coherent system $\cdot k$-out-of- $n$ system $\cdot$ Discrete lifetime distribution . Reliability · Order statistics

Mathematics Subject Classification $62 \mathrm{~N} 05 \cdot 62 \mathrm{E} 15 \cdot 60 \mathrm{~K} 10$

\section{Introduction}

Coherent systems are of special importance in reliability theory since they have been widely used to model mathematically sophisticated technical devices composed of simple elements. The system is said to be coherent if its structure function is increasing in every component and such that each component is relevant (a component is irrelevant if it does not matter whether or not it is working). The classical monograph here is Barlow and Proschan (1975). Important examples for coherent systems are $k$-out-of- $n$ systems. The $k$-out-of- $n$ system works as long as at least $k$ of its components work.

Krzysztof Jasiński

krzys@mat.umk.pl

1 Faculty of Mathematics and Computer Science, Nicolaus Copernicus University, Chopina 12/18, 87-100 Torun, Poland 
The cases $k=1$ and $k=n$ correspond to parallel and series systems, respectively. During the last few years, the properties of coherent systems have been studied quite extensively in the literature, see for example Navarro and Burkschat (2011), Eryilmaz (2013), Kelkinnama et al. (2015), Nair et al. (2018), Ashrafi et al. (2018), Hazra and Finkelstein (2019), Kelkinnama and Asadi (2019), and the references therein.

In real life situations, we may have only partial information on the status of the system or its components. Based on this partial information many authors paid their attention to the residual lifetime and inactivity time of coherent systems, especially $k$-out-of- $n$ systems. For more details and recent studies, we refer the reader to Tavangar (2016), Navarro et al. (2017), Eryilmaz and Bayramoglu (2018), Navarro and Calì (2019) and Goli (2019). Most results in this area have been obtained under the assumption that the component lifetimes are absolutely continuous random variables (rvs). The analysis is much easier than in the discrete case, where there are possible ties between component failures with non-zero probability. However, the discrete models occur very often, for example, when the system performs a task repetitively and its components have certain probabilities of breakdown upon each cycle or when the component lifetimes represent the numbers of turn-on and switch-off up to failures. Reliability properties of coherent systems composed of components with discrete lifetimes have been investigated by Weiss (1962), Young (1970), Tank and Eryilmaz (2015), Dembińska (2018), Dembińska et al. (2021), Davies and Dembińska (2019) and Dembińska and Goroncy (2020). Dembińska and Jasiński (2020) studied maximum likelihood estimation based on discrete component lifetimes of a $k$-out-of- $n$ system. Navarro et al. (2008), Miziuła and Rychlik (2014) or Eryilmaz et al. (2016) considered the systems with arbitrary lifetime distributions which can be discrete in particular.

For coherent systems, among other things, the authors have been interesting in the number of working components while the system is still working, see Eryilmaz (2010). In contrast, Ross et al. (1980) focused on the number of component failures in systems whose component lifetimes are exchangeable. Asadi and Berred (2012) determined the probability that there are exactly $i, i=0, \ldots, n-k$, failures in the $k$-out-of- $n$ system under the condition that it is operating at time $t$ and the component lifetimes are independent and identically distributed (IID) absolutely continuous rvs. Several properties of this probability were considered. Further, these results were extended to the coherent systems. Our aim is to compute this probability in the case of $k$-outof- $n$ systems and next coherent systems consisting of $n$ components whose discrete lifetimes are possibly dependent and not necessarily identically distributed (DNID) rvs. This was done in Sections 2 and 3, respectively. Moreover, we will prove that the formulas obtained in the most popular case, that is in the model of IID rvs, correspond to the respective ones derived by Asadi and Berred (2012).

Throughout the paper we use the following notation. Let $\mathcal{P}^{n}$ denote the set of all permutations $\left(j_{1}, j_{2}, \ldots, j_{n}\right)$ of $(1,2, \ldots, n)$, and $\mathcal{P}_{s}^{n}$ stand for the subset of $\mathcal{P}^{n}$ consisting only of permutations satisfying

$$
j_{1}<j_{2}<\cdots<j_{s}, \quad j_{s+1}<j_{s+2}<\cdots<j_{n} .
$$




\section{Results on failed components in $k$-out-of-n systems}

Consider a $k$-out-of- $n$ system composed of $n$ components whose discrete lifetimes $T_{1}, \ldots, T_{n}$ are allowed to be DNID rvs having cumulative distribution functions (cdfs) $F_{i}(t)=\mathrm{P}\left(T_{i} \leq t\right), i=1, \ldots, n$, and taking values in finite or infinite subsets of the set of non-negative integers. Then $T_{1: n} \leq \ldots \leq T_{n: n}$ stand for the respective order statistics. Since a $k$-out-of- $n$ system functions as long as at least $k$ of their components function, its lifetime $T_{k, n}$ is the $(n-k+1)$ th smallest of the component lifetimes, i.e. $T_{k, n}=T_{n-k+1: n}$.

Let us denote by $N_{k, n}(t)$ the number of failed components of a $k$-out-of- $n$ system at time $t$. We assume that at time $t$ the system is still working, i.e. $T_{k, n}>t$. It is of interest to determine the following conditional probability

$$
p_{t}(i, k, n)=\mathrm{P}\left(N_{k, n}(t)=i \mid T_{k, n}>t\right), \quad i=0,1, \ldots, n-k .
$$

Observe that

$$
S_{k, n}(t)=n-N_{k, n}(t)
$$

describes the number of working components of a used system at time $t$. Therefore studying $N_{k, n}(t)$ and $S_{k, n}(t)$ is equivalent.

To compute $p_{t}(i, k, n)$, note that the event $\left\{N_{k, n}(t)=i\right\}$ takes place if and only if the event $\left\{T_{i: n} \leq t<T_{i+1: n}\right\}$ occurs. Then

$$
\begin{aligned}
p_{t}(i, k, n) & =\frac{\mathrm{P}\left(N_{k, n}(t)=i, T_{k, n}>t\right)}{\mathrm{P}\left(T_{k, n}>t\right)}=\frac{\mathrm{P}\left(T_{i: n} \leq t<T_{i+1: n}, T_{n-k+1: n}>t\right)}{\mathrm{P}\left(T_{n-k+1: n}>t\right)} \\
& =\frac{\mathrm{P}\left(T_{i: n} \leq t<T_{i+1: n}\right)}{\mathrm{P}\left(T_{n-k+1: n}>t\right)}, \quad i=0,1, \ldots, n-k .
\end{aligned}
$$

By (1) we see at once that we need a method of dealing with order statistics corresponding to discrete rvs which are DNID. Dembińska (2018) obtained the formula for the probability of the event $\left\{T_{i: n} \leq t, T_{i+1: n}>t\right\}$

$$
\mathrm{P}\left(T_{i: n} \leq t, T_{i+1: n}>t\right)=\sum_{\left(j_{1}, \ldots, j_{n}\right) \in \mathcal{P}_{i}^{n}} \mathrm{P}\left({ }^{\left(j_{1}, \ldots, j_{n}\right)} H_{i}^{t}\right),
$$

where

$$
{ }^{\left(j_{1}, \ldots, j_{n}\right)} H_{i}^{t}=\left(\bigcap_{l=1}^{i}\left\{T_{j_{l}} \leq t\right\}\right) \cap\left(\bigcap_{l=i+1}^{n}\left\{T_{j_{l}}>t\right\}\right) .
$$

Knowing the dependence structure between $T_{1}, \ldots, T_{n}$, we can give the simplified forms of (2). Under the exchangeability assumption, the system components have identical distributions, but they affect one another within the system. If $T_{1}, \ldots, T_{n}$ are exchangeable, that is for any $\left(j_{1}, \ldots, j_{n}\right) \in \mathcal{P}^{n}$, the random vector $\left(T_{j_{1}}, \ldots, T_{j_{n}}\right)$ has the same distribution as $\left(T_{1}, \ldots, T_{n}\right)$ or if $T_{1}, \ldots, T_{n}$ are independent, then we have 


$$
\begin{aligned}
& \mathrm{P}\left(T_{i: n} \leq t, T_{i+1: n}>t\right) \\
& =\left\{\begin{array}{cl}
\left(\begin{array}{c}
n \\
i
\end{array}\right) \mathrm{P}\left(\left(\bigcap_{l=1}^{i}\left\{T_{l} \leq t\right\}\right) \cap\left(\bigcap_{l=i+1}^{n}\left\{T_{l}>t\right\}\right)\right), & \text { if } T_{1}, \ldots, T_{n} \text { are exchangeable, } \\
\sum_{\left(j_{1}, \ldots, j_{n}\right) \in \mathcal{P}_{i}^{n}}\left(\prod_{l=1}^{i} F_{j_{l}}(t)\right)\left(\prod_{l=i+1}^{n} \bar{F}_{j_{l}}(t)\right), & \text { if } T_{1}, \ldots, T_{n} \text { are independent. }
\end{array}\right.
\end{aligned}
$$

Using (3), Davies and Dembińska (2019) proposed the representation of the reliability function of $T_{k, n}$

$$
\mathrm{P}\left(T_{k, n}>t\right)=P\left(T_{n-k+1: n}>t\right)=\sum_{s=0}^{n-k} \sum_{\left(j_{1}, \ldots, j_{n}\right) \in \mathcal{P}_{s}^{n}} \mathrm{P}\left({ }^{\left(j_{1}, \ldots, j_{n}\right)} H_{s}^{t}\right) .
$$

As before, expression (5) has the closed-forms in particular cases. Thus

$$
\begin{aligned}
& \mathrm{P}\left(T_{n-k+1: n}>t\right) \\
& = \begin{cases}\sum_{s=0}^{n-k}\left(\begin{array}{l}
n \\
s
\end{array}\right) \mathrm{P}\left(\left(\bigcap_{l=1}^{s}\left\{T_{l} \leq t\right\}\right) \cap\left(\bigcap_{l=s+1}^{n}\left\{T_{l}>t\right\}\right)\right), & \text { if } T_{1}, \ldots, T_{n} \text { are exchangeable, } \\
\sum_{s=0}^{n-k} \sum_{\left.j_{1}, \ldots, j_{n}\right) \in \mathcal{P}_{s}^{n}}\left(\prod_{l=1}^{s} F_{j_{l}}(t)\right)\left(\prod_{l=s+1}^{n} \bar{F}_{j_{l}}(t)\right), & \text { if } T_{1}, \ldots, T_{n} \text { are independent. }\end{cases}
\end{aligned}
$$

Combining (2) with (5) and (4) with (6), we prove the following result.

Theorem 1 Consider a k-out-of-n system consisting of $n$ elements whose discrete lifetimes $T_{1}, \ldots, T_{n}$ are assumed to be DNID rvs. Then for any $i=0, \ldots, n-k$, we get

$$
p_{t}(i, k, n)=\frac{\sum_{\left(j_{1}, \ldots, j_{n}\right) \in \mathcal{P}_{i}^{n}} \mathrm{P}\left({ }^{\left(j_{1}, \ldots, j_{n}\right)} H_{i}^{t}\right)}{\sum_{s=0}^{n-k} \sum_{\left(j_{1}, \ldots, j_{n}\right) \in \mathcal{P}_{s}^{n}} \mathrm{P}\left(\left(j_{1}, \ldots, j_{n}\right) H_{s}^{t}\right)},
$$

where ${ }^{\left(j_{1}, \ldots, j_{n}\right)} H_{v}^{t}$ are defined in (3). In particular, we have

$$
\begin{aligned}
& p_{t}(i, k, n) \\
& =\left\{\begin{array}{l}
\frac{\left(\begin{array}{l}
n \\
i
\end{array}\right) \mathrm{P}\left(\left(\bigcap_{l=1}^{i}\left\{T_{l} \leq t\right\}\right) \cap\left(\bigcap_{l=i+1}^{n}\left\{T_{l}>t\right\}\right)\right)}{\sum_{s=0}^{n-k}\left(\begin{array}{l}
n \\
s
\end{array}\right) \mathrm{P}\left(\left(\bigcap_{l=1}^{s}\left\{T_{l} \leq t\right\}\right) \cap\left(\bigcap_{l=s+1}^{n}\left\{T_{l}>t\right\}\right)\right)}, \\
\frac{\sum_{\left(j_{1}, \ldots, j_{n}\right) \in \mathcal{P}_{i}^{n}}\left(\prod_{l=1}^{i} F_{j_{l}}(t)\right)\left(\prod_{l=i+1}^{n} \bar{F}_{j_{l}}(t)\right)}{\sum_{s=0}^{n-k} \sum_{\left(j_{1}, \ldots, j_{n}\right) \in \mathcal{P}_{s}^{n}}\left(\prod_{l=1}^{s} F_{j_{l}}(t)\right)\left(\prod_{l=s+1}^{n} \bar{F}_{j_{l}}(t)\right)},
\end{array}\right.
\end{aligned}
$$


Example 1 Let us consider a system that consists of four components which are at risk for failure in a discrete manner. This implies that the lifetimes are discretely distributed. During such a lifetime, there are cycles, and in each cycle, there is a shock to the $i$ th component, $i=1,2,3,4$, which it survives with probability $p \in(0,1)$. Moreover, in each cycle there is a shock to all the four components, which all of them survive with probability $\theta \in(0,1)$ and neither of them survives with probability $1-\theta$. The events of surviving the different shocks are independent of each other and from cycle to cycle. When a component fails, it remains forever inoperative.

Let $T_{i}, i=1,2,3,4$ represent the number of cycles up to and including the failure of the $i$ th component. Then, the survival function of the random vector $\left(T_{1}, T_{2}, T_{3}, T_{4}\right)$ has the form

$$
\begin{aligned}
P\left(T_{1}>t_{1}, T_{2}>t_{2}, T_{3}>t_{3}, T_{4}>t_{4}\right)= & p^{t_{1}+t_{2}+t_{3}+t_{4}} \theta^{\max \left\{t_{1}, t_{2}, t_{3}, t_{4}\right\}}, \\
& t_{1}, t_{2}, t_{3}, t_{4}=0,1,2, \ldots
\end{aligned}
$$

The distribution of $\left(T_{1}, T_{2}, T_{3}, T_{4}\right)$ is a special case of the multivariate geometric distribution proposed by Esary and Marshall (1973). Note that $T_{1}, T_{2}, T_{3}, T_{4}$ are exchangeable and dependent.

Additionally, we assume that the system operate as long as at least 3 of its components function. Then $T_{3,4}=T_{2: 4}$. By (9) we get

$$
\begin{aligned}
P & \left(T_{1} \leq t, T_{2}>t, T_{3}>t, T_{4}>t\right) \\
= & \sum_{x=1}^{t}\left[P\left(T_{1}>x-1, T_{2}>t, T_{3}>t, T_{4}>t\right)\right. \\
& \left.\quad-P\left(T_{1}>x, T_{2}>t, T_{3}>t, T_{4}>t\right)\right]=p^{3 t} \theta^{t}\left(1-p^{t}\right), \quad t=1,2, \ldots
\end{aligned}
$$

Now combining (8) with (9) and (10), after simple algebra, we obtain

$$
\begin{aligned}
p_{t}(0,3,4) & =\frac{P\left(T_{1}>t, T_{2}>t, T_{3}>t, T_{4}>t\right)}{P\left(T_{1}>t, T_{2}>t, T_{3}>t, T_{4}>t\right)+4 P\left(T_{1} \leq t, T_{2}>t, T_{3}>t, T_{4}>t\right)} \\
& =\frac{p^{t}}{4-3 p^{t}}, \\
p_{t}(1,3,4) & =\frac{4 P\left(T_{1} \leq t, T_{2}>t, T_{3}>t, T_{4}>t\right)}{P\left(T_{1}>t, T_{2}>t, T_{3}>t, T_{4}>t\right)+4 P\left(T_{1} \leq t, T_{2}>t, T_{3}>t, T_{4}>t\right)} \\
& =\frac{4-4 p^{t}}{4-3 p^{t}} .
\end{aligned}
$$

Corollary 1 In the case when the discrete lifetimes $T_{1}, \ldots, T_{n}$ are IID rvs with a common distribution function $F$, the probability in (7) can be simplified to

$$
p_{t}(i, k, n)=\frac{\left(\begin{array}{c}
n \\
i
\end{array}\right) F^{i}(t) \bar{F}^{n-i}(t)}{\sum_{s=0}^{n-k}\left(\begin{array}{c}
n \\
s
\end{array}\right) F^{s}(t) \bar{F}^{n-s}(t)}=\frac{\left(\begin{array}{c}
n \\
i
\end{array}\right)(\phi(t))^{i}}{\sum_{s=0}^{n-k}\left(\begin{array}{c}
n \\
s
\end{array}\right)(\phi(t))^{s}}, \quad i=0, \ldots, n-k,
$$


where $\phi(t)=\frac{F(t)}{\bar{F}(t)}$.

Remark 1 The formula given in (11) is the same as that obtained by Asadi and Berred (2012), who considered the model of IID continuous rvs. Therefore several properties proposed by Asadi and Berred (2012) are also valid in the discrete case.

\section{Results on failed components in coherent systems}

Our aim is to extend the results obtained in Section 2 to other coherent systems than $k$-out-of- $n$ structures. Let us consider a coherent system composed of $n$ elements numbered $1, \ldots, n$. We will denote by $T$ the system lifetime. Then $T_{1}, \ldots, T_{n}$ are its discrete component lifetimes which are assumed to be DNID. Here there is a chance that a number of components in the operating system may have already failed, but the failure times are unknown. Hence, it is natural to ask what is the probability that there are $i, i=0, \ldots, n-1$, failures in the system under the condition that it is still working at time $t$, i.e.

$$
p_{t}^{c}(i, n)=\mathrm{P}\left(T_{i: n} \leq t<T_{i+1: n} \mid T>t\right)=\frac{\mathrm{P}\left(T_{i: n} \leq t<T_{i+1: n}, T>t\right)}{\mathrm{P}(T>t)}
$$

for $i=0, \ldots, n-1$, which is the extended version of the probability (1). We start with recalling relevant concepts and facts.

We say that $P \subset\{1, \ldots, n\}$ is a path set of a coherent system if it operates when all the elements with indices in $P$ work. A path set is said to be minimal if it is a minimal set of components whose functioning ensures the functioning of the system. Then the lifetime $T$ can be represented as

$$
T=\max _{1 \leq j \leq s} \min _{p \in P_{j}} T_{p}
$$

where $P_{1}, \ldots, P_{s}$ are the minimal paths sets, see Barlow and Proschan (1975, p. 13). This means that a system works if all the components in one of its paths work. In the case of a $k$-out-of- $n$ system, there are $\left(\begin{array}{l}n \\ k\end{array}\right)$ minimal path sets, namely, all of the sets consisting of exactly $k$ components. By the representation (12), Navarro et al. (2007, Theorem 3.1) expressed the reliability function of $T$ as

$$
P(T>t)=\sum_{j=1}^{s}(-1)^{j+1} \sum_{1 \leq k_{1}<\cdots<k_{j} \leq s} \mathrm{P}\left(\bigcap_{p \in P_{k_{1}} \cup \cdots \cup P_{k_{j}}}\left\{T_{p}>t\right\}\right) .
$$

We also apply (12) to determine the probability of the event $\left\{T_{i: n} \leq t<T_{i+1: n}, T>\right.$ $t\}$ as follows 


$$
\begin{aligned}
\mathrm{P}\left(T_{i: n} \leq t<T_{i+1: n}, T>t\right) & =\mathrm{P}\left(T_{i: n} \leq t<T_{i+1: n}, \max _{1 \leq j \leq s} \min _{p \in P_{j}} T_{p}>t\right) \\
& =\mathrm{P}\left(T_{i: n} \leq t<T_{i+1: n}, \bigcup_{j=1}^{s}\left\{\min _{p \in P_{j}} T_{p}>t\right\}\right) \\
& =\mathrm{P}\left(\bigcup_{j=1}^{s}\left\{T_{i: n} \leq t<T_{i+1: n}, \min _{p \in P_{j}} T_{p}>t\right\}\right) .
\end{aligned}
$$

Further, using the inclusion-exclusion formula, we deduce that

$$
\begin{aligned}
\mathrm{P} & \left.T_{i: n} \leq t<T_{i+1: n}, T>t\right) \\
& =\sum_{j=1}^{s}(-1)^{j+1} \sum_{1 \leq k_{1}<\cdots<k_{j} \leq s} \mathrm{P}\left(T_{i: n} \leq t<T_{i+1: n}, \bigcap_{l=1}^{j}\left\{\min _{p \in P_{k_{l}}} T_{p}>t\right\}\right) \\
& =\sum_{j=1}^{s}(-1)^{j+1} \sum_{1 \leq k_{1}<\cdots<k_{j} \leq s} \mathrm{P}\left(T_{i: n} \leq t<T_{i+1: n}, \min _{p \in P_{k_{1}} \cup \cdots \cup P_{k_{j}}} T_{p}>t\right) \\
& =\sum_{j=1}^{s}(-1)^{j+1} \sum_{1 \leq k_{1}<\cdots<k_{j} \leq s} \mathrm{P}\left(T_{i: n} \leq t<T_{i+1: n}, \bigcap_{p \in P_{k_{1}} \cup \cdots \cup P_{k_{j}}}\left\{T_{p}>t\right\}\right) .
\end{aligned}
$$

Combining (14) with (13), we are ready to state the following theorem.

Theorem 2 Consider a coherent system composed of $n$ components. We assume that the discrete component lifetimes $T_{1}, \ldots, T_{n}$ are DNID rvs. Then for any $i=0, \ldots, n-$ 1 , we have

$$
p_{t}^{c}(i, n)=\frac{\sum_{j=1}^{s}(-1)^{j+1} \sum_{1 \leq k_{1}<\cdots<k_{j} \leq s} \mathrm{P}\left(T_{i: n} \leq t<T_{i+1: n}, \bigcap_{p \in P_{k_{1}} \cup \cdots \cup P_{k_{j}}}\left\{T_{p}>t\right\}\right)}{\sum_{j=1}^{s}(-1)^{j+1} \sum_{1 \leq k_{1}<\cdots<k_{j} \leq s} \mathrm{P}\left(\bigcap_{p \in P_{k_{1}} \cup \cdots \cup P_{k_{j}}}\left\{T_{p}>t\right\}\right)} .
$$

Under the assumption that $T_{1}, \ldots, T_{n}$ are exchangeable, Navarro et al. (2008) proposed that the reliability function of $T$ can be written as a mixture of the reliability functions of the associated order statistics. Thus

$$
P(T>t)=\sum_{m=1}^{n} s_{m} \mathrm{P}\left(T_{m: n}>t\right),
$$

where $s_{m} \geq 0, m=1, \ldots, n$ and $\sum_{m=1}^{n} s_{m}=1$. They generalized the earlier results established by Samaniego (1985) and Navarro and Rychlik (2007). The vector $\mathbf{s}=$ 
$\left(s_{1}, \ldots, s_{n}\right)$ is called the Samaniego signature and it depends only on the structure of the system and is independent of the distribution of $\left(T_{1}, \ldots, T_{n}\right)$. The formula in (16) can be equivalently rewritten as

$$
\mathrm{P}(T>t)=\sum_{m=1}^{n} \alpha_{m} \mathrm{P}\left(T_{1: m}>t\right)
$$

where

$$
\alpha_{m}=\sum_{j=1}^{s}(-1)^{j+1} \sum_{1 \leq k_{1}<\cdots<k_{s} \leq j} \mathrm{I}\left(\left|\bigcup_{l=1}^{j} P_{k_{l}}\right|=m\right), m=1, \ldots, n,
$$

where $\left|\bigcup_{l=1}^{j} P_{k_{l}}\right|$ denotes the cardinality of each $\bigcup_{l=1}^{j} P_{k_{l}}$ and $\sum_{m=1}^{n} \alpha_{m}=1$, see for details Dembińska and Goroncy (2020, p. 19). The vector $\alpha=\left(\alpha_{1}, \ldots, \alpha_{n}\right)$ is called a minimal signature of a system. This notation was introduced and exploited by Navarro et al. (2007). Now combining (17) with (18), we get

$$
\begin{aligned}
& P(T>t) \\
& = \begin{cases}\sum_{m=1}^{n} P\left(\bigcap_{l=1}^{m}\left\{X_{l}>t\right\}\right) \sum_{j=1}^{s}(-1)^{j+1} \sum_{1 \leq k_{1}<\cdots<k_{s} \leq j} & \mathrm{I}\left(\left|\bigcup_{l=1}^{j} P_{k_{l}}\right|=m\right), \\
& \text { if } T_{1}, \ldots, T_{n} \text { are exchangeable, } \\
\sum_{m=1}^{n}\left(\prod_{l=1}^{m} \bar{F}_{l}(t)\right) \sum_{j=1}^{s}(-1)^{j+1} \sum_{1 \leq k_{1}<\cdots<k_{s} \leq j} & \text { I }\left(\left|\bigcup_{l=1}^{j} P_{k_{l}}\right|=m\right), \\
& \text { if } T_{1}, \ldots, T_{n} \text { are independent. }\end{cases}
\end{aligned}
$$

In these particular cases we obtain the simplified forms of the numerator of (15). Since

$$
\begin{aligned}
\left\{T_{i: n} \leq t<T_{i+1: n}, \bigcap_{p \in P_{k_{1}} \cup \ldots \cup P_{k_{j}}}\left\{T_{p}>t\right\}\right\} & \left\{\bigcup _ { | \bigcup _ { l = 1 } ^ { j } P _ { k _ { l } } | = 1 } ^ { n - i } \left\{\text { exactly }\left|\bigcup_{l=1}^{j} P_{k_{l}}\right| \text { of } T_{p}, p \in \bigcup_{l=1}^{j} P_{k_{l}} \text {, are }>t,\right.\right. \\
& \text { exactly } i \text { of } n-\left|\bigcup_{l=1}^{j} P_{k_{l}}\right| \text { of } T_{p} \text { are } \leq t, \\
& \text { and the rest } \left.n-i-\left|\bigcup_{l=1}^{j} P_{k_{l}}\right| \text { of } T_{p} \text { are }>t\right\},
\end{aligned}
$$


we obtain

$$
\begin{gathered}
\mathrm{P}\left(T_{i: n} \leq t<T_{i+1: n}, \bigcap_{p \in P_{k_{1}} \cup \cdots \cup P_{k_{j}}}\left\{T_{p}>t\right\}\right)=\sum_{m=1}^{n-i} \mathrm{I}\left(\left|\bigcup_{l=1}^{j} P_{k_{l}}\right|=m\right) \\
\quad \sum_{\left(j_{1}, \cdots, j_{n-m}\right) \in \mathcal{P}_{i}^{n-m}} P\left(\bigcap_{l=1}^{m}\left\{T_{p_{l}}>t\right\} \cap \bigcap_{l=1}^{i}\left\{T_{j_{l}} \leq t\right\} \cap \bigcap_{l=i+1}^{n-m}\left\{T_{j_{l}}>t\right\}\right),
\end{gathered}
$$

where $p_{1}, \ldots, p_{m} \in P_{k_{1}} \cup \cdots \cup P_{k_{j}}$ and $\left\{j_{1}, \ldots, j_{n-m}\right\}=\{1, \ldots, n\}-\left\{p_{1}, \ldots, p_{m}\right\}$. Hence

$$
\begin{aligned}
& \mathrm{P}\left(T_{i: n} \leq t<T_{i+1: n}, \bigcap_{p \in P_{k_{1}} \cup \cdots \cup P_{k_{j}}}\left\{T_{p}>t\right\}\right) \\
& \int \sum_{m=1}^{n-i} \mathrm{I}\left(\left|\bigcup_{l=1}^{j} P_{k_{l}}\right|=m\right)\left(\begin{array}{c}
n-m \\
i
\end{array}\right) P\left(\bigcap_{l=1}^{m}\left\{T_{p_{l}}>t\right\} \cap \bigcap_{l=1}^{i}\left\{T_{j_{l}} \leq t\right\} \cap \bigcap_{l=i+1}^{n-m}\left\{T_{j_{l}}>t\right\}\right), \\
& =\left\{\begin{array}{c}
\text { if } T_{1}, \ldots, T_{n} \text { are exchangeable, } \\
\sum_{m=1}^{n-i} \mathrm{I}\left(\left|\bigcup_{l=1}^{j} P_{k_{l}}\right|=m\right) \prod_{l=1}^{m} \bar{F}_{p_{l}}(t) \sum_{\left(j_{1}, \ldots, j_{n-m}\right) \in \mathcal{P}_{i}^{n-m}} \prod_{l=1}^{i} F_{j_{l}}(t) \prod_{l=i+1}^{n-m} \bar{F}_{j_{l}}(t), \\
\text { if } T_{1}, \ldots, T_{n} \text { are independent. }
\end{array}\right.
\end{aligned}
$$

Corollary 2 Combining (20) with (19) we get the closed-forms of (15), when $T_{1}, \ldots, T_{n}$ are exchangeable or independent not necessarily identically distributed, respectively.

Remark 2 The same proofs of Theorem 2 and the formulas (19) and (20) still go when we drop the assumption that $T_{1}, \ldots, T_{n}$ are the discrete rvs. Therefore Theorem 2 and Corollary 2 can be applied not only in the discrete case but also in the general situation of any distribution of component lifetimes.

In the model of IID rvs Theorem 2 specializes in the following result.

Theorem 3 Suppose that the discrete component lifetimes $T_{1}, \ldots, T_{n}$ are IID rvs with a common distribution function $F$. Then for any $i=0, \ldots, n-1$, we have

$$
p_{t}^{c}(i, n)=\frac{F^{i}(t) \bar{F}^{n-i}(t) \sum_{j=1}^{s}(-1)^{j+1} \sum_{1 \leq k_{1}<\cdots<k_{j} \leq s} \sum_{m=1}^{n-i} \mathrm{I}\left(\left|\bigcup_{l=1}^{j} P_{k_{l}}\right|=m\right)\left(\begin{array}{c}
n-m \\
i
\end{array}\right)}{\sum_{m=1}^{n} \bar{F}^{m}(t) \sum_{j=1}^{s}(-1)^{j+1} \sum_{1 \leq k_{1}<\cdots<k_{s} \leq j} \mathrm{I}\left(\left|\bigcup_{l=1}^{j} P_{k_{l}}\right|=m\right)},
$$


or equivalently by (16)

$$
p_{t}^{c}(i, n)=\frac{F^{i}(t) \bar{F}^{n-i}(t) \sum_{j=1}^{s}(-1)^{j+1} \sum_{1 \leq k_{1}<\cdots<k_{j} \leq s} \sum_{m=1}^{n-i} \mathrm{I}\left(\left|\bigcup_{l=1}^{j} P_{k_{l}}\right|=m\right)\left(\begin{array}{c}
n-m \\
i
\end{array}\right)}{\sum_{w=0}^{n-1}\left(\sum_{m=w+1}^{n} s_{m}\right)\left(\begin{array}{l}
n \\
w
\end{array}\right) F^{w}(t) \bar{F}^{n-w}(t)} .
$$

Corollary 3 In the case of k-out-of-n systems, the probability (15) reduces to (7) and (21), (22) to (11), respectively.

Asadi and Berred (2012) computed the probability $p_{t}^{c}(i, n)$ in the case of a coherent system consisting of $n$ components whose lifetimes are assumed to be IID, where $F$ is continuous. Denoted by $\tilde{S}_{w}=\sum_{m=w+1}^{n} s_{m}, 0 \leq w \leq n-1$, they got

$$
p_{t}^{c}(i, n)=\frac{\left(\begin{array}{c}
n \\
i
\end{array}\right) F^{i}(t) \bar{F}^{n-i}(t) \tilde{S}_{i}}{\sum_{w=0}^{n-1} \tilde{S}_{w}\left(\begin{array}{c}
n \\
w
\end{array}\right) F^{w}(t) \bar{F}^{n-w}(t)}=\frac{\left(\begin{array}{c}
n \\
i
\end{array}\right) \phi^{i}(t) \tilde{S}_{i}}{\sum_{w=0}^{n-1} \tilde{S}_{w}\left(\begin{array}{c}
n \\
w
\end{array}\right) \phi^{w}(t)} .
$$

We will show that the above formula is equivalent to that obtained in (22). Studying (23) and (22) it suffices to check the equality between the numerators. Comparing (16) with (17) Dembińska and Goroncy (2020) determined the minimal signature from the corresponding Samaniego signature as follows

$$
\alpha_{m}=\left(\begin{array}{l}
n \\
m
\end{array}\right) \sum_{r=n-m+1}^{n} s_{r}(-1)^{r-1-n+m}\left(\begin{array}{c}
m-1 \\
n-r
\end{array}\right), \quad m=1, \ldots, n .
$$

Thus we can derive the formulas for $s_{m}, m=1, \ldots, n$ in terms of $\alpha_{1}, \ldots, \alpha_{n}$. It follows that

$$
s_{m}=\sum_{r=1}^{n-m+1} \frac{\left(\begin{array}{c}
n-m \\
r-1
\end{array}\right)}{\left(\begin{array}{l}
n \\
r
\end{array}\right)} \alpha_{r}, \quad m=1, \ldots, n \text {. }
$$

Using (24), we get

$$
\tilde{S}_{i}=\sum_{m=i+1}^{n} s_{m}=\sum_{m=i+1}^{n}\left[\sum_{r=1}^{n-m+1} \frac{\left(\begin{array}{l}
n-i \\
r-1
\end{array}\right)}{\left(\begin{array}{l}
n \\
r
\end{array}\right)} \alpha_{r}\right]=\sum_{m=1}^{n-i}\left[\frac{1}{\left(\begin{array}{l}
n \\
m
\end{array}\right)} \sum_{r=i+1}^{n-m+1}\left(\begin{array}{c}
n-r \\
m-1
\end{array}\right)\right] \alpha_{m} .
$$

Combining $\sum_{r=i+1}^{n-m+1}\left(\begin{array}{c}n-r \\ m-1\end{array}\right)=\left(\begin{array}{c}n-i \\ m\end{array}\right)$ (see Feller 1957, p. 64) with (18), we have

$$
\tilde{S}_{i}=\sum_{m=1}^{n-i}\left[\frac{1}{\left(\begin{array}{c}
n \\
m
\end{array}\right)}\left(\begin{array}{c}
n-i \\
m
\end{array}\right) \sum_{j=1}^{s}(-1)^{j+1} \sum_{1 \leq k_{1}<\cdots<k_{s} \leq j} \mathrm{I}\left(\left|\bigcup_{l=1}^{j} P_{k_{l}}\right|=m\right)\right] .
$$

Now putting (25) to the numerator of (23) and using the equality $\frac{\left(\begin{array}{c}n \\ i\end{array}\right)\left(\begin{array}{c}n-i \\ m\end{array}\right)}{\left(\begin{array}{c}n \\ m\end{array}\right)}=\left(\begin{array}{c}n-m \\ i\end{array}\right)$, we obtain the numerator of (22). Thus the probability $p_{t}^{c}(i, n)$ can be computed by 
the same formula in the case when the component lifetimes $T_{1}, \ldots, T_{n}$ are IID either continuous or discrete rvs.

Remark 3 Using (25) we obtain the following formula for the Samaniego signature of the system in terms of its minimal path sets. For $1 \leq i \leq n$,

$$
s_{i}=\tilde{S}_{i-1}-\tilde{S}_{i}=\sum_{m=1}^{n-i+1} \frac{\left(\begin{array}{c}
n-i \\
m-1
\end{array}\right)}{\left(\begin{array}{c}
n \\
m
\end{array}\right)} \sum_{j=1}^{s}(-1)^{j+1} \sum_{1 \leq k_{1}<\cdots<k_{j} \leq s} I\left(\left|\bigcup_{w=1}^{j} P_{k_{w}}\right|=m\right)
$$

Example 2 Consider a coherent system with the lifetime

$$
T=\min \left\{T_{1}, T_{2}, \max \left\{T_{3}, T_{4}\right\}\right\},
$$

where $T_{i}, i=1,2,3,4$, are assumed to be independent rvs such that $T_{i}$ has a geometric distribution $g e o\left(p_{i}\right), i=1,2,3,4$, where $p_{1}=p_{3}=p \in(0,1)$ and $p_{2}=p_{4}=\theta \in$ $(0,1), p \neq \theta$. Hence in this example we have rvs of two different types: $T_{1}, T_{3} \sim F_{1}$ and $T_{2}, T_{4} \sim F_{2}$, where

$F_{1}(x)=1-(1-p)^{t}, \quad \bar{F}_{1}(t)=(1-p)^{t}, \quad F_{2}(x)=1-(1-\theta)^{t}, \quad \bar{F}_{2}(t)=(1-\theta)^{t}$,

for $t=0,1, \ldots$ This coherent system has two minimal path sets, namely, $P_{1}=$ $\{1,2,3\}, P_{2}=\{1,2,4\}$. By Corollary 2 we obtain

$$
\begin{aligned}
& p_{t}^{c}(0,4)=\frac{\bar{F}_{1}^{2}(t) \bar{F}_{2}^{2}(t)}{\bar{F}_{1}^{2}(t) \bar{F}_{2}(t)+\bar{F}_{1}(t) \bar{F}_{2}^{2}(t)-\bar{F}_{1}^{2}(t) \bar{F}_{2}^{2}(t)}=\frac{1}{(1-p)^{-t}+(1-\theta)^{-t}-1}, \\
& p_{t}^{c}(1,4)=\frac{F_{2}(t) \bar{F}_{1}^{2}(t) \bar{F}_{2}(t)+F_{1}(t) \bar{F}_{2}^{2}(t) \bar{F}_{1}(t)}{\bar{F}_{1}^{2}(t) \bar{F}_{2}(t)+\bar{F}_{1}(t) \bar{F}_{2}^{2}(t)-\bar{F}_{1}^{2}(t) \bar{F}_{2}^{2}(t)}=\frac{(1-p)^{-t}+(1-\theta)^{-t}-2}{(1-p)^{-t}+(1-\theta)^{-t}-1},
\end{aligned}
$$

for $t=0,1, \ldots$. Moreover, $p_{t}^{c}(2,4)=p_{t}^{c}(3,4)=0$ because of the structure of the system.

If $p=\theta$ that is when $T_{1}, T_{2}, T_{3}, T_{4}$ are IID rvs, we get

$$
\begin{aligned}
& p_{t}^{c}(0,4)=\frac{1}{2(1-p)^{-t}-1}, \\
& p_{t}^{c}(1,4)=\frac{2(1-p)^{-t}-2}{2(1-p)^{-t}-1}, \quad t=0,1, \ldots
\end{aligned}
$$

Alternatively, the formulas given in (27) can be obtained by (23). It suffices to use the Samaniego signature of the system with the lifetime (26) which has the form $s=\left(\frac{1}{2}, \frac{1}{2}, 0,0\right)$, see Shaked and Suarez-Llorens (2003) for more details. 
Now we assume that the component lifetimes $T_{1}, T_{2}, T_{3}, T_{4}$ are the rvs as in Example 1. By Corollary 2, after simple algebra, we obtain

$$
\begin{aligned}
& p_{t}^{c}(0,4) \\
& \quad=\frac{P\left(T_{1}>t, T_{2}>t, T_{3}>t, T_{4}>t\right)}{P\left(T_{1}>t, T_{2}>t, T_{3}>t\right)+P\left(T_{1}>t, T_{2}>t, T_{4}>t\right)-P\left(T_{1}>t, T_{2}>t, T_{3}>t, T_{4}>t\right)} \\
& \quad=\frac{p^{t}}{2-p^{t}}, \\
& p_{t}^{c}(1,4) \\
& \quad=\frac{P\left(T_{1}>t, T_{2}>t, T_{3}>t, T_{4} \leq t\right)+P\left(T_{1}>t, T_{2}>t, T_{4}>t, T_{3} \leq t\right)}{P\left(T_{1}>t, T_{2}>t, T_{3}>t\right)+P\left(T_{1}>t, T_{2}>t, T_{4}>t\right)-P\left(T_{1}>t, T_{2}>t, T_{3}>t, T_{4}>t\right)} \\
& \quad=\frac{2-2 p^{t}}{2-p^{t}},
\end{aligned}
$$

for $t=0,1, \ldots$

\section{Summary and conclusions}

Engineers and system designers are of interest to maintain the system in optimum working condition. Therefore they need to determine the number of spares that should be available in the depot for this purpose. This problem is important because the failure and unavailability of the system may cause high unexpected costs to the potential users.

Coherent systems play an important role in various fields of applications. Depending on the type of utilization, each technical system has a specific design or structure. The whole device can operate even if a number of its elements have already failed. However, if the number of failed components passes a certain threshold, then the system does fail. Hence, the computation of the probability of the number of failed components in the system, proposed in this paper, is a useful quantity. This would allow the operators of the systems for greater planning and more efficient use of resources. The probability provides crucial information for preventing the system's failure. The system operators can try to change or to restore a failed component to an operative state to avoid or to diminish the occurrence of the system failure. These actions are very important to establish optimal designs of production systems, telecommunication networks, supply chains, etc.

Funding Not applicable.

Availability of data and material Not applicable.

\section{Declarations}

Conflict of interest The author states that there is no conflict of interest.

Code availability Not applicable. 
Open Access This article is licensed under a Creative Commons Attribution 4.0 International License, which permits use, sharing, adaptation, distribution and reproduction in any medium or format, as long as you give appropriate credit to the original author(s) and the source, provide a link to the Creative Commons licence, and indicate if changes were made. The images or other third party material in this article are included in the article's Creative Commons licence, unless indicated otherwise in a credit line to the material. If material is not included in the article's Creative Commons licence and your intended use is not permitted by statutory regulation or exceeds the permitted use, you will need to obtain permission directly from the copyright holder. To view a copy of this licence, visit http://creativecommons.org/licenses/by/4.0/.

\section{References}

Asadi M, Berred A (2012) On the number of failed components in a coherent operating system. Stat Probab Lett 82:2156-2163

Ashrafi S, Zarezadeh S, Asadi M (2018) Reliability modeling of coherent systems with shared components based on sequential order statistics. J Appl Probab 55:845-861

Barlow RE, Proschan F (1975) Statistical theory of reliability and life testing: probability models. Holt, Rinehart and Winston, New York

Davies K, Dembińska A (2019) On the number of failed components in a $k$-out-of- $n$ system upon system failure when the lifetimes are discretely distributed. Reliab Eng Syst Saf 188:47-61

Dembińska A (2018) On reliability analysis of $k$-out-of- $n$ systems consisting of heterogeneous components with discrete lifetimes. IEEE Trans Reliab 67:1071-1083

Dembińska A, Goroncy A (2020) Moments of order statistics from DNID discrete random variables with application in reliability. J Comput Appl Math 371:112703

Dembińska A, Jasiński K (2020) Maximum likelihood estimators based on discrete component lifetimes of a k-out-of-n system. Test. https://doi.org/10.1007/s11749-020-00724-0

Dembińska A, Nikolov NI, Stoimenova E (2021) Reliability properties of $k$-out-of- $n$ systems with one cold standby unit. J Comput Appl Math 388:113289

Eryilmaz S (2010) Number of working components in consecutive $k$-out-of- $n$ system while it is working. Commun Stat Simul Comput 39:683-692

Eryilmaz S (2013) On reliability of a $k$-out-of- $n$ system equipped with a single warm standby component. IEEE Trans Reliab 62:499-503

Eryilmaz S, Bayramoglu K (2018) Residual lifetime of consecutive k-out-of-n systems under double monitoring. IEEE Trans Reliab 61:792-797

Eryilmaz S, Koutras MV, Triantatyllou JS (2016) Mixed three-state $k$-out-of- $n$ systems under double monitoring. IEEE Trans Reliab 61:792-797

Esary JD, Marshall AW (1973) Multivariate geometric distributions generated by a cumulative damage process. Techinal Report 55\#Y73041A. Naval Postgraduate School, Monterey, California

Feller W (1957) An introduction to probability theory and its applications, vol I, 2nd edn. Wiley, New York

Goli S (2019) On the conditional residual lifetime of coherent systems under double regularly checking. Naval Res Logist 66:352-363

Hazra NK, Finkelstein M (2019) Comparing lifetimes of coherent systems with dependent components operating in random environments. J Appl Probab 56:937-957

Kelkinnama M, Asadi M (2019) Stochastic and ageing properties of coherent systems with dependent identically distributed components. Stat Papers 60:455-471

Kelkinnama M, Tavangar M, Asadi M (2015) New developments on stochastic properties of coherent systems. IEEE Trans Reliab 64:1276-86

Miziuła P, Rychlik T (2014) Sharp bounds for lifetime variances of reliability systems with exchangeable components. IEEE Trans Reliab 63:850-857

Nair NU, Sankaran PG, Balakrishnan N (2018) Reliability modelling and analysis in discrete time. Academic Press, London

Navarro J, Burkschat M (2011) Coherent systems based on sequential order statistics. Naval Res Logist 58:123-135

Navarro J, Calì C (2019) Inactivity times of coherent systems with dependent components under periodical inspections. Appl Stoch Models Bus Ind 35:871-892 
Navarro J, Longobardi M, Pellerey F (2017) Comparison results for inactivity times of k-out-of-n and general coherent systems with dependent components. Test 26:822-846

Navarro J, Ruiz JM, Sandoval CJ (2007) Properties of coherent systems with dependent components. Commun Stat Theory Methods 36:175-191

Navarro J, Rychlik T (2007) Reliability and expectation bounds for coherent systems with exchangeable components. J Multivar Anal 98:102-113

Navarro J, Samaniego FJ, Balakrishnan N, Bhattacharya D (2008) On the application and extension of system signatures in engineering reliability. Naval Res Logist 55:313-327

Ross SM, Shahshahani M, Weiss G (1980) On the number of component failures in systems whose component lives are exchangeable. Math Oper Res 5:358-365

Samaniego FJ (1985) On closure of the IFR class under formation of coherent systems. IEEE Trans Reliab 34:69-72

Shaked M, Suarez-Llorens A (2003) On the comparison of reliability experiments based on the convolution order. J Am Stat Assoc 98:693-702

Tank F, Eryilmaz S (2015) The distributions of sum, minima and maxima of generalized geometric random variables. Stat Pap 56:1191-1203

Tavangar M (2016) Conditional inactivity time of components in a coherent operating system. IEEE Trans Reliab 65:359-369

Weiss G (1962) On certain redundant systems which operate at discrete times. Technometrics 4:169-174

Young D (1970) The order statistics of the negative binomial distribution. Biometrika 57:181-186

Publisher's Note Springer Nature remains neutral with regard to jurisdictional claims in published maps and institutional affiliations. 